\title{
TABAGISMO ENTRE ALUNOS E FUNCIONÁRIOS DA FACULDADE DE SAÚDE PÚBLICA DA UNIVERSIDADE DE SÃO PAULO*
}

\author{
Antonio Pedro Mirra**

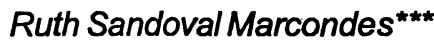 \\ José Maria Pacheco de Souza ${ }^{\star \star \star \star \star}$ \\ Glacilda Telles Menezes Stewien ${ }^{\star \star \star \star \star}$
}

\begin{abstract}
RESUMO: Dois inquéritos sobre a prevalência do tabagismo entre os alunos e funcionários da Faculdade de Saúde Pública da Universidade de São Paulo, presentes à aula inaugural, cujo tema foi tabagismo, foram realizados em 1980 e 1995 . No primeiro inquérito, em 1980, estavam presentes 177 funcionários e alunos de cursos de especialização e pós graduação (homens $=38,4 \%$ e mulheres $=61,6 \%$ ) e 128 , no segundo, em 1995 (homens $=29,7 \%$ e mulheres $=70,3 \%$ ). Observou-se que houve um decréscimo na prevalência de fumantes regulares de $56,9 \%$ para $26,3 \%$ no sexo masculino e de $45,9 \%$ para $22,2 \%$ no feminino, sendo essas diferenças mais acentuadas entre os mais jovens (20 a 39 anos de idade). Os profissionais com formação biológica foram os mais motivados para não adquirirem o vício de fumar, seguindo-se o grupo com formação básica em ciências exatas e sociais e os educadores; os profissionais relacionados às letras, advogados, foram os que não mostraram motivação, pois houve aumento da prevalência de fumantes regulares. A idade no inicio de fumar foi semelhante à da população geral (10 - 14 anos de idade), bem como as razões para esse início e a manutenção do vício. A grande maioria dos entrevistados afirmou ter conhecimento dos malefícios do tabaco. A midia falada (TVI Rádio) foi selecionada como meio mais adequado para a divulgação dos efeitos nocivos do fumo. Programas de combate ao tabagismo mais efetivos devem ser implementados na escolas de saúde pública, a fim de que ocorra a redução de fumantes entre os alunos dos seus cursos regulares e seus funcionários.
\end{abstract}

PALAVRAS-CHAVE: tabagismo, prevalência, escola de saúde pública

\footnotetext{
* Trabalho realizado na Faculdade de Saúde Pública da USP.

**Coordenador do Registro de Câncer de São Paulo, do Departamento de Epidemiologia da Faculdade de Saúde Pública, USP. Chefe da Divisão de Registros de Câncer da Fundação Oncocentro de São Paulo.

***Professora Titular, aposentada, do Departamento de Prática de Saúde Pública da Faculdade de Saúde Pública, USP.

****Professor Titular do Departamento de Epidemiologia da Faculdade de Saúde Pública, USP.

*****Professora Doutora, aposentada, do Departamento de Prática de Saúde Pública da Faculdade de Saúde Pública, USP.Faculdade de Saúde Pública da USP End: Av. Dr. Arnaldo, 715 - $1^{\circ}$ andar CEP: 012046-904 São Paulo Tel : 3066-7799 Fax: 282-2920
} 


\section{INTRODUÇÃO}

O tabagismo é considerado um problema de saúde pública, porquanto são atribuídos a esse vício $90 \%$ dos casos de câncer de pulmão, $86 \%$ de bronquite e enfisema, $25 \%$ dos processos isquêmicos do coração e $30 \%$ dos cânceres extrapulmonares. Embora seja a maior causa isolada evitável de doença e morte, ocorrem no mundo 3 milhões de óbitos ( $5 \%$ da mortalidade geral) e, no Brasil, 80 a 100 mil mortes (10,9\% da mortalidade geral), anualmente. Em nosso país, 30 milhões de indivíduos com mais de 15 anos de idade são fumantes ( $32,6 \%$ da população), sendo $44,4 \%$ mulheres e dois terços moradores de zonas urbanas (3).

Nos programas de combate ao tabagismo, os profissionais de saúde têm papel muito importante junto à população por sua atuação em ações educativas, a fim de influir no decréscimo do número de pessoas fumantes e dos que se iniciam no tabagismo.

Inquéritos realizados entre estudantes de medicina da Universidade de São Paulo (Ribeirão Preto e São Paulo), Faculdade de Ciências Médicas da Santa Casa de Misericórdia de São Paulo, Organização Santamarense de Educação e Cultura - OSEC (atual Universidade Santo Amaro), Faculdade de Ciências Médicas de Sorocaba (PUC), Faculdade de Medicina de Bragança Paulista e Universidade Federal do Rio Grande do Sul (Porto Alegre), na década de 80 , mostraram que a prevalência de fumantes decresceu de $41,3 \%$ para $12,1 \%$ entre os homens e de $34,3 \%$ para $11,2 \%$ entre as mulheres $(1,2,4,5,6,7,8)$.

Programas informativos sobre tabagismo devem ser introduzidos nas escolas de saúde pública em suas atividades curriculares a fim de que os seus alunos venham a constituir uma população livre do tabaco e sejam participantes ativos na luta anti - tabágica.

O objetivo deste trabalho é apresentar os resultados de dois inquéritos realizados sobre a prevalência de fumantes entre alunos e funcionários da Faculdade de Saúde Pública da Universidade de São Paulo, nos anos de 1980 e 1995.

\section{MATERIAL E MÉTODOS}

Em 1980 e 1995 foram realizados inquéritos sobre a prevalência do tabagismo entre os alunos e funcionários da Faculdade de Saúde Pública da Universidade de São 
Paulo presentes à aula inaugural, cujo tema foi tabagismo. Utilizou-se um questionário para a obtenção das informações.

Estavam presentes alunos que freqüentavam cursos de especialização e pósgraduação nesses anos e funcionários: 177 no inquérito de 1980 (sexo masculino = 68 $38,4 \%$ e feminino $=109-61,6 \%$ ) e 128 no inquérito de 1995 (sexo masculino $=38-29,7 \%$ e feminino $=90-70,3 \%$ ).

A distribuição dos alunos e funcionários segundo o ano, sexo e idade é mostrada na tabela 1. A tabela 2 mostra a distribuição dos alunos e funcionários segundo o ano, sexo e grupo de profissões.

\section{RESULTADOS}

A distribuição dos alunos e funcionários dos sexos masculino e feminino fumantes, não fumantes e ex-fumantes, segundo o ano e idade, é dada pelas tabelas 3 e 4 . No inquérito referente ao ano de 1980 constatou-se, nos sexos masculino e feminino, $56,9 \%$ (I.C.:44,7\% - $68,6 \%$ ) e $45,9 \%$ (I.C.:36,3\% - 55,7\%) de fumantes regulares respectivamente, enquanto que, no inquérito de 1995, se observou uma queda desses indices para 26,3\% (I.C.: 13,4\% $-43,1 \%$ ) e $22,2 \%$ (I.C.: 14,1\% - 32,2\%). Considerando-se os grupos etários, houve diferenças significativas para os grupos $30-39$ anos do sexo masculino $\left(x^{2}=7,08 ; p=0,01\right)$ e $20-29$ anos $\left(x^{2}=4,04 ; p=0,04\right)$ e $30-39$ anos $\left(x^{2}=9,12 ; p=0,00\right)$ do sexo feminino.

Nas tabelas 5 e 6 tem-se a distribuição dos alunos e funcionários fumantes, nãofumantes e ex-fumantes, segundo o ano, sexo e grupo de profissões. No inquérito de 1980, no sexo masculino houve uma maior prevalência de fumantes no grupo de profissões abrangendo médicos e dentistas (43,8\%), engenheiros, veterinários, advogados $(61,5 \%)$ e educadores (33,3\%). Entre os funcionários, com $64,3 \%$, tem-se o grupo de encanadores, marceneiros, pintores, gráficos, motoristas, técnicos de aparelhos, serventes e continuos. O outro grupo presente foi o de técnicos de administração, escriturários, secretárias, tesoureiros, bibliotecários e estatísticos $(50,0 \%)$. No sexo feminino, a maior prevalência de fumantes ocorreu no grupo de médicas e dentistas (75,0\%); enfermagem (60,0\%); assistentes sociais, sociólogas, pedagogas e visitadoras sanitárias (41,2\%); terapeutas ocupacionais e nutricionistas (50,0\%); entre as funcionárias, tem-se o grupo de costureiras e serventes (50,0\%). 
No inquérito de 1995 houve um decréscimo em muitos grupos de profissões em ambos os sexos. No sexo masculino, tem-se médicos e dentistas $-28,6 \%$; engenheiros, agrônomos, veterinários - 36,8\% ; houve ausência de fumantes nos grupos de técnicos de administração e educadores. No sexo feminino, houve decréscimo dos índices entre os grupos de médicas e dentistas - 30,8\% ; fisioterapeutas, terapeutas ocupacionais, fonoaudiólogas, psicólogas e nutricionistas - 33,3\% e educadoras - 30,0\%. No grupo de engenheiras, agrônomas, veterinárias, geólogas, advogadas, entretanto, houve aumento de fumantes $(50,0 \%)$.

$\mathrm{Na}$ tabela 7 tem-se a distribuição dos alunos e funcionários fumantes e ex-fumantes, segundo o ano, sexo e idade do início de fumar. Verifica-se que o início do fumar ocorreu a partir do grupo 10 - 14 anos de idade, não havendo diferenças significativas em todas as idades e em ambos os inquéritos. $\left(x^{2}=1,39 ; p=0,24\right.$ e $\left.x^{2}=0,91 ; p=0,34\right)$.

A distribuição dos alunos e funcionários fumantes e ex-fumantes, segundo o ano, sexo e número de cigarros fumados por dia é verificada na tabela 8 ; não houve diferenças significativas em ambos os sexos e nos inquéritos de 1980 e 1995 ( $x^{2}=1,89 ; p=0,39$ e $x^{2}$ $=0,34 ; p=0,84)$.

Em ambos os inquéritos, verificou-se que os entrevistados fumavam quando se apresentavam felizes, alegres, nervosos, tristes e ociosos em $\quad 50,9 \%$ em 1980 e $56,9 \%$ dos casos em 1995. A grande maioria dos entrevistados, em ambos os inquéritos, fumava cigarros com filtro $(90,9 \%)$.

Entre as razões que levaram a iniciar o vicio, em ambos os inquéritos, predominaram a curiosidade $(45,7 \%$ e $46,6 \%$ ) e outros colegas que fumavam (35,3\% e 32,8\%). Entretanto, entre as razões que os fazem fumar atualmente está o prazer $(60,7 \%$ e $80,0 \%)$, seguindose a sensação de calma $(15,7 \%$ e $10,0 \%)$ e colegas que fumam $(5,6 \%$ e $3,3 \%)$.

Quando indagados se tinham informações sobre os maleficios do tabaco, 93,8\% e 95,3\% responderam terem conhecimento, bem como sabiam da existência de parentes que morreram de câncer de pulmão e outras doenças pulmonares $(38,4 \%$ e $55,5 \%)$ e infarto do miocárdio $(40,7 \%$ e $50,8 \%)$.

Entre os meios que devem ser utilizados para a divulgação dos malefícios do tabagismo, selecionaram TV/ Rádio $(48,1 \%$ e $66,4 \%)$ e palestras de especialistas $(40,8 \%$ e $38,3 \%$ ). 


\section{DISCUSSÃo}

Os profissionais de saúde têm uma participação de destaque nos programas de combate ao tabagismo, principalmente pela sua atuação em ações educativas junto à população. As informações sobre fumo que são levadas a este grupo de profissionais, bem como à prática de não fumar, revestem-se de importância muito grande para o sucesso dos programas anti-tabaco.

As escolas de saúde pública têm um papel importante neste mister, pois recebem em seus cursos de especialização e pós-graduação profissionais que têm atividades diversificadas na área da saúde comunitária, como ocorre na Faculdade de Saúde Pública da Universidade de São Paulo.

Nos inquéritos sobre prevalência do tabagismo entre os alunos e funcionários da Faculdade de Saúde Pública da USP, realizados em 1980 e 1995, observou-se que houve um decréscimo de fumantes regulares, em ambos os sexos, de $56,9 \%$ para $26,3 \%$ (homens) e de $45,9 \%$ para $22,2 \%$ (mulheres), sendo que estas diferenças foram mais acentuadas entre os mais jovens, nos grupos etários de 30-39 anos, no sexo masculino e $20-29$ anos e $30-39$ anos, no feminino.

O comportamento dos alunos e funcionários, em relação ao vício de fumar, não foi idêntico, considerando-se sua profissão. Os profissionais com formação básica biológica são os mais motivados a não adquirirem o vício, possivelmente, por manterem uma relação mais direta com os pacientes, permitindo uma observação mais nítida dos malefícios do tabaco no organismo humano. Aqueles profissionais de formação básica em ciências exatas e sociais e os educadores tiveram uma sensibilização para o problema, porém, em menor escala. $\mathrm{O}$ grupo de profissionais que não teve motivação foi aquele relacionado às letras, advogados, em especial as mulheres, em que houve um aumento da prevalência de fumantes regulares.

Em relação à idade do início de fumar, o comportamento dos alunos e funcionários foi semelhante ao que ocorre na população geral, 10-14 anos de idade. Igualmente, em relação às razões para iniciar e manter o vício de fumar, houve semelhança com a população geral.

Embora um dos objetivos de todo programa de combate ao fumo seja o de levar informações sobre os malefícios do tabaco, com o surgimento de grande número de doenças 
tabaco-relacionadas, nem sempre esse aspecto causa a motivação esperada, em relação ao vício de fumar. Entre os alunos e funcionários em ambos os inquéritos, o conhecimento dessas doenças atingindo parentes próximos, embora conhecedores dos prejuízos do fumo, não foi razão suficiente para influir no início ou no deixar de fumar.

A influência da propaganda intensa utilizada pela indústria do tabaco, através da TV e rádio, em que grandes investimentos monetários são feitos para levar crianças, jovens e mulheres à prática de fumar, aliás com sucesso, também deve ser usada no combate ao tabagismo. Nos inquéritos realizados, a mídia falada e as palestras de especialistas foram os melhores meios selecionados para a divulgação dos efeitos nocivos do tabaco, com real possibilidade de sucesso.

Programas de combate ao tabagismo mais efetivos devem ser implementados nas escolas de saúde pública, pois, quando informações são levadas aos alunos e funcionários, como ocorreu na motivação dos inquéritos realizados em 1980 e 1995, podem levar a resultados apreciáveis na redução da prevalência de fumantes regulares.

\section{REFERÊNCIAS BIBLIOGRÁFICAS}

RIGATTO, M. et al. Influência do curso médio da UFRGS sobre seus alunos em relação ao tabagismo. In: Semana do Hospital de Clínicas de Porto Alegre. Anais. Porto Alegre, 1989.

RODRIGUES, E.M.S. Tabagismo entre estudantes da USP, Campus RibeirãoPreto - SP, no Período de 1980/1988. Ribeirão Preto, 1989. [Tese de Mestrado - Faculdade de Medicina de Ribeirão Preto da USP]

ROSEMBERG, J. Tabagismo. $2^{a}$ ed. São Paulo, Almed/Edusp, 1988. Série Problema de Saúde Pública.

ROSEMBERG, J. \& PERON, S. Tabagismo entre estudantes da Faculdade de Ciências Médicas de Sorocaba: tabagismo nos acadêmicos de medicina e nos médicos. J. Pneumol., 16:13-22, 1990.

RUFFINO NETTO, A. et al. Tabagismo entre acadêmicos de Ribeirão Preto. J.Pneumol., 7:164-70, 1981. 
RUFFINO NETTO, A. et al. Tendência do tabagismo entre acadêmicos de Ribeirão Preto, SP; estudo prospectivo. J. Pneumol., 14:163-9, 1988.

RUFFINO NETTO, A. et al Hábito de fumar entre acadêmicos ligados à área da saúde: alguns aspectos econômicos, Ribeirão Preto. Medicina (Ribeirão Preto), 22:95-104, 1989.

SZEGO, T., BECHARA, M.J. \& RODRIGUES, J.C. Levantamento epidemiológico sobre o vício de fumar entre estudantes de medicina no Estado de São Paulo. Rev. Ass. Med. Bras., 31:13-6, 1985. 


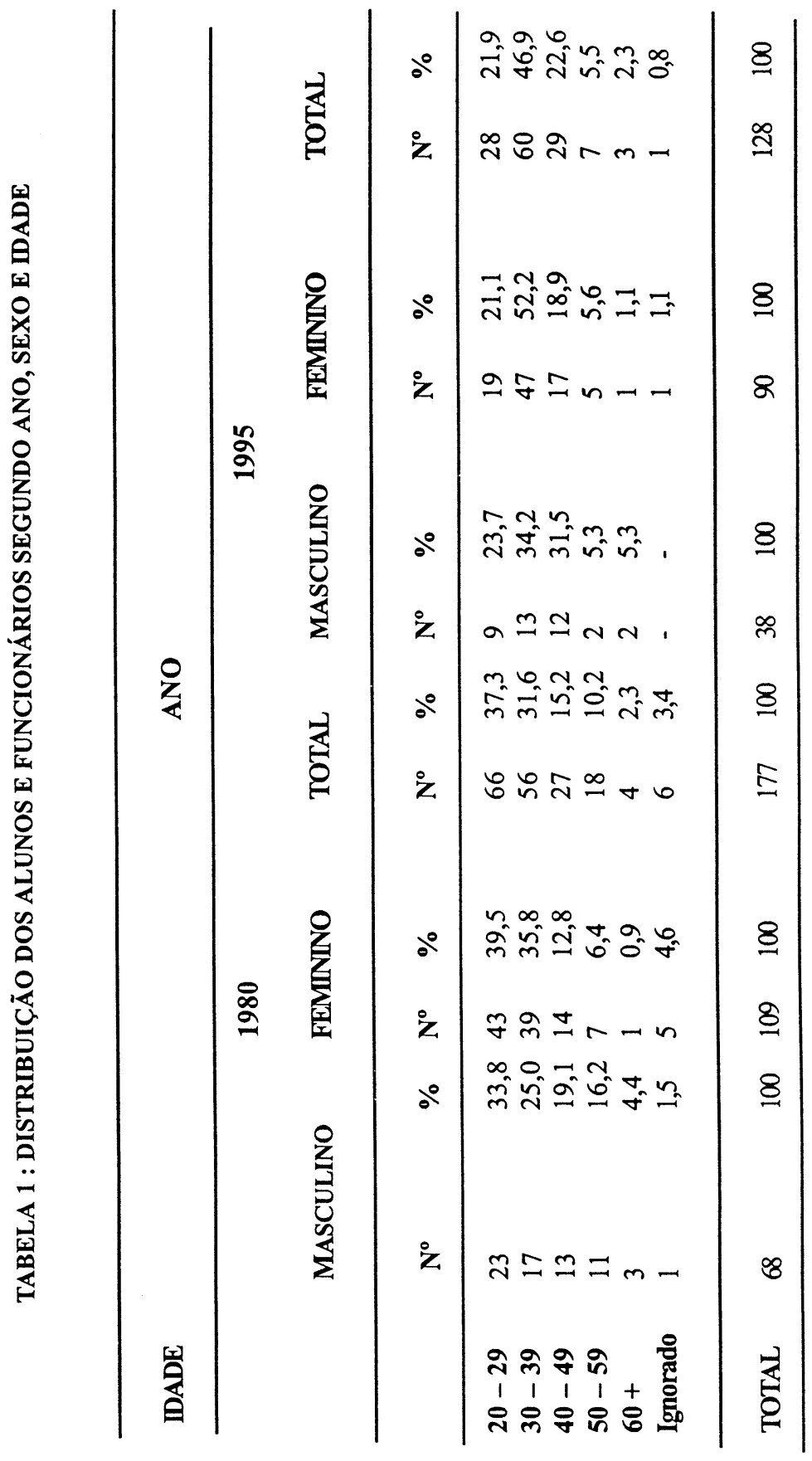




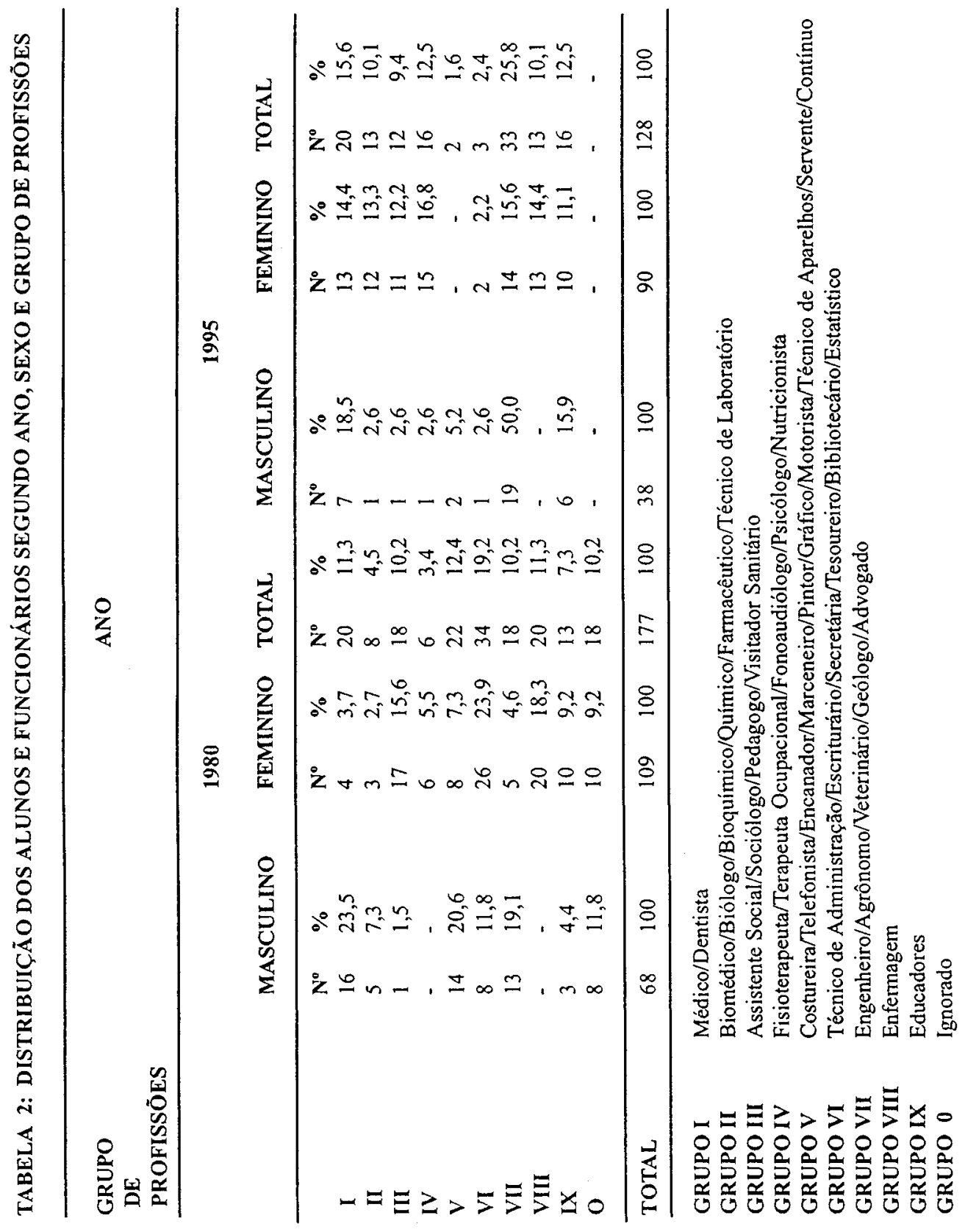




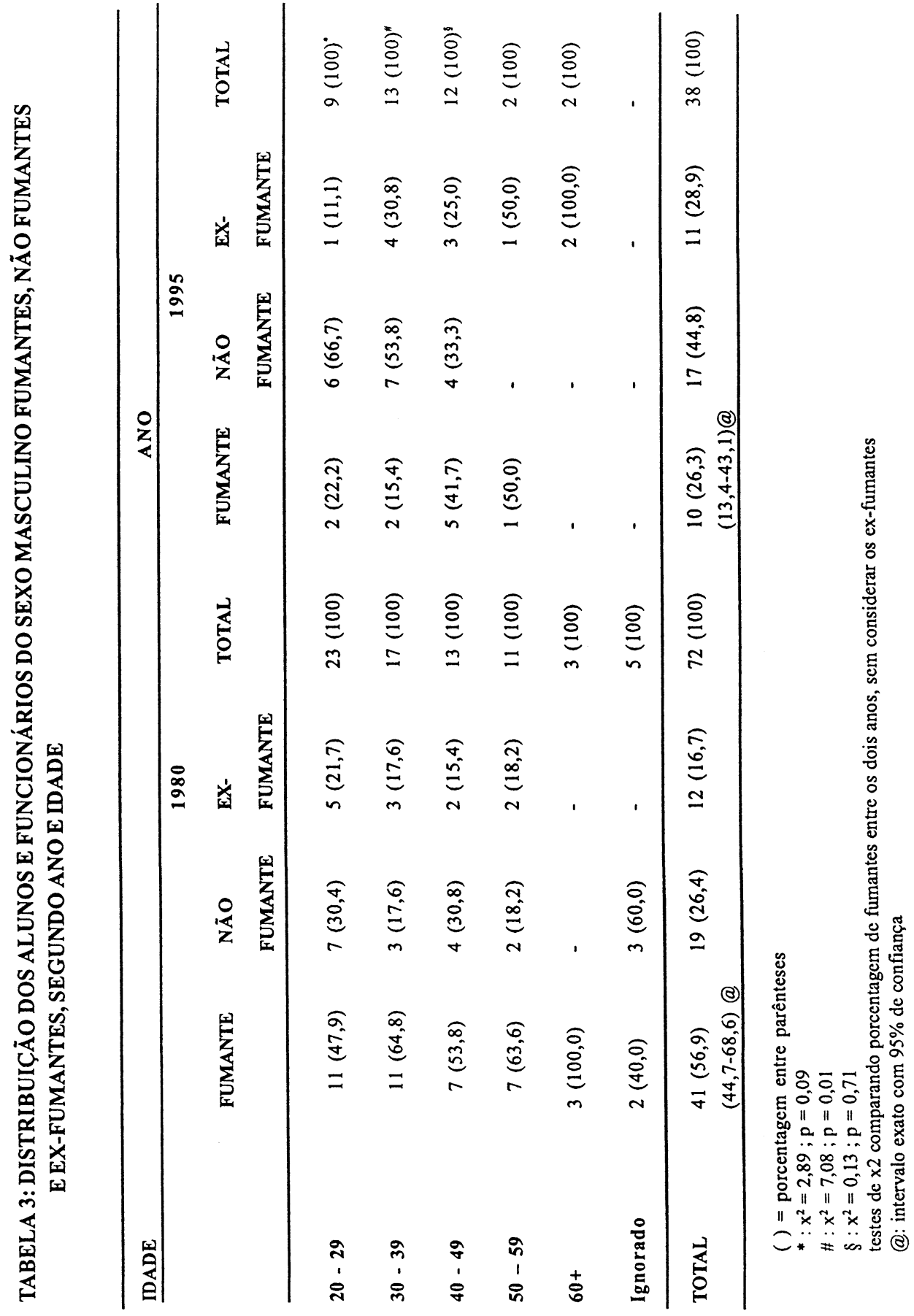




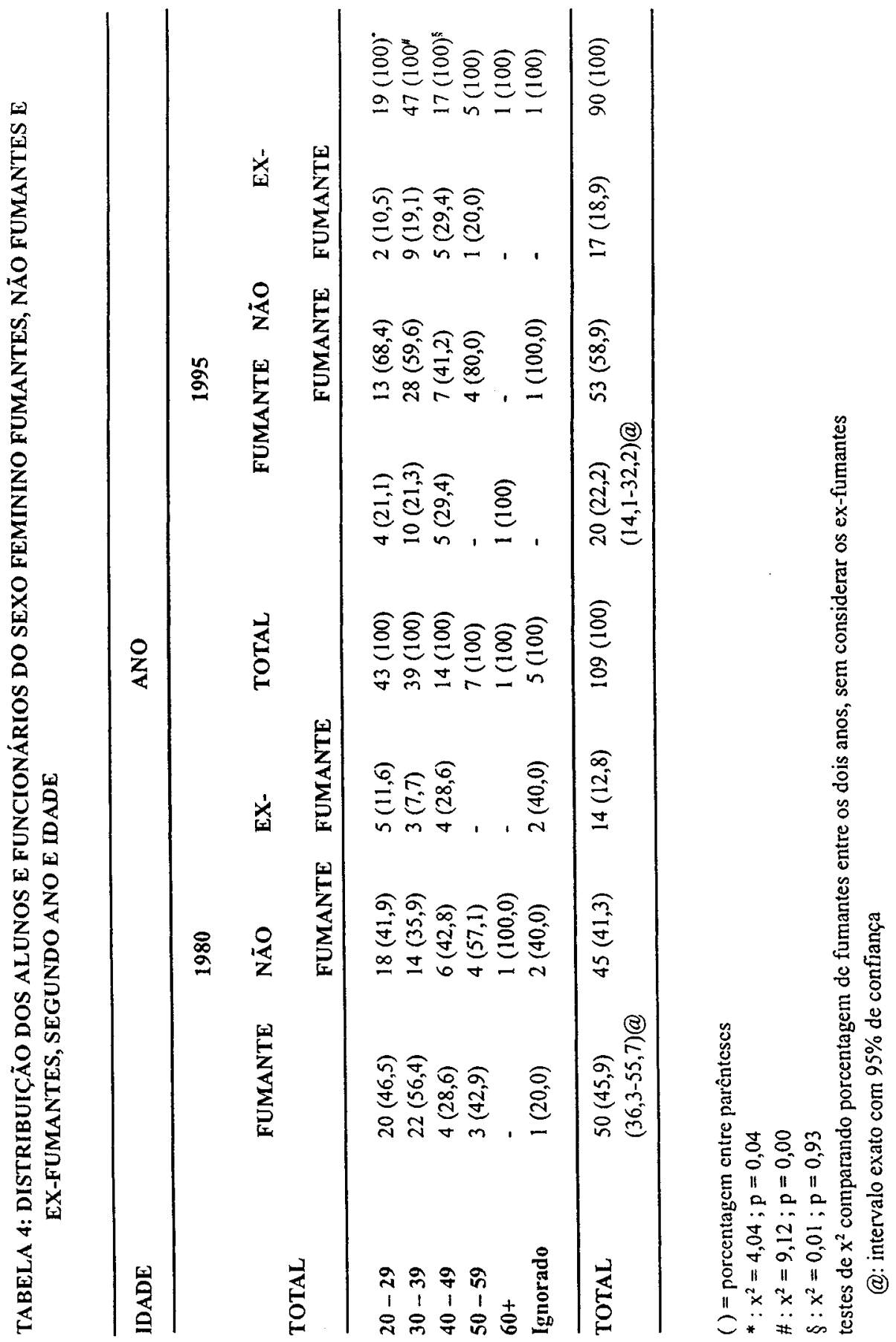




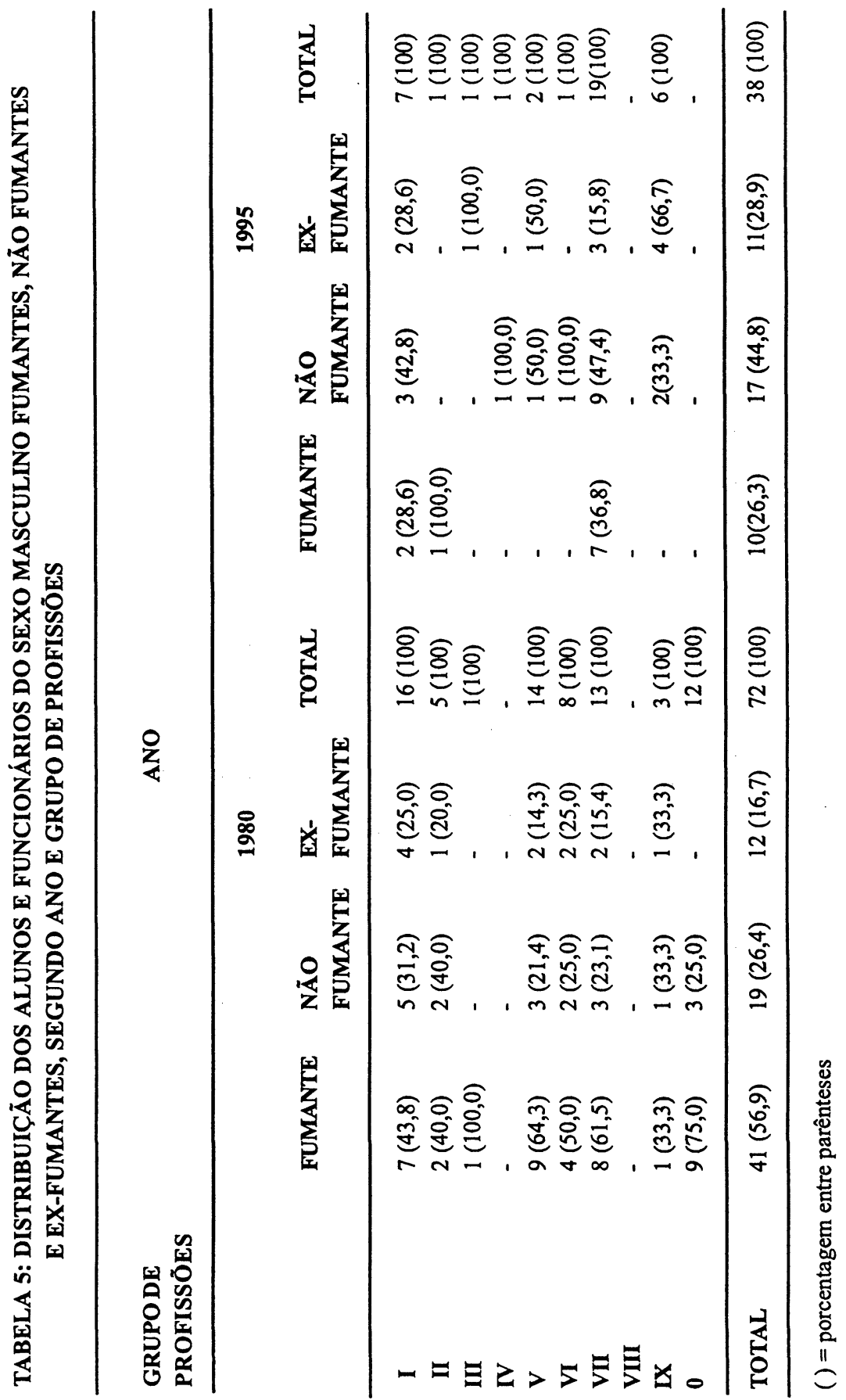




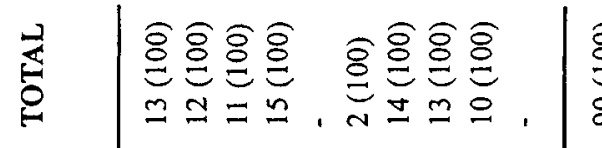

응 2

ป气

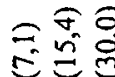

Nmm, I N

结 क

$\geq \sum$

ñ

$n \infty \infty N, N \in=$

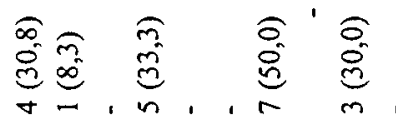

용

$\frac{3}{6}$

으ํํㅇ으으응

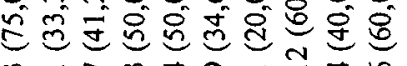




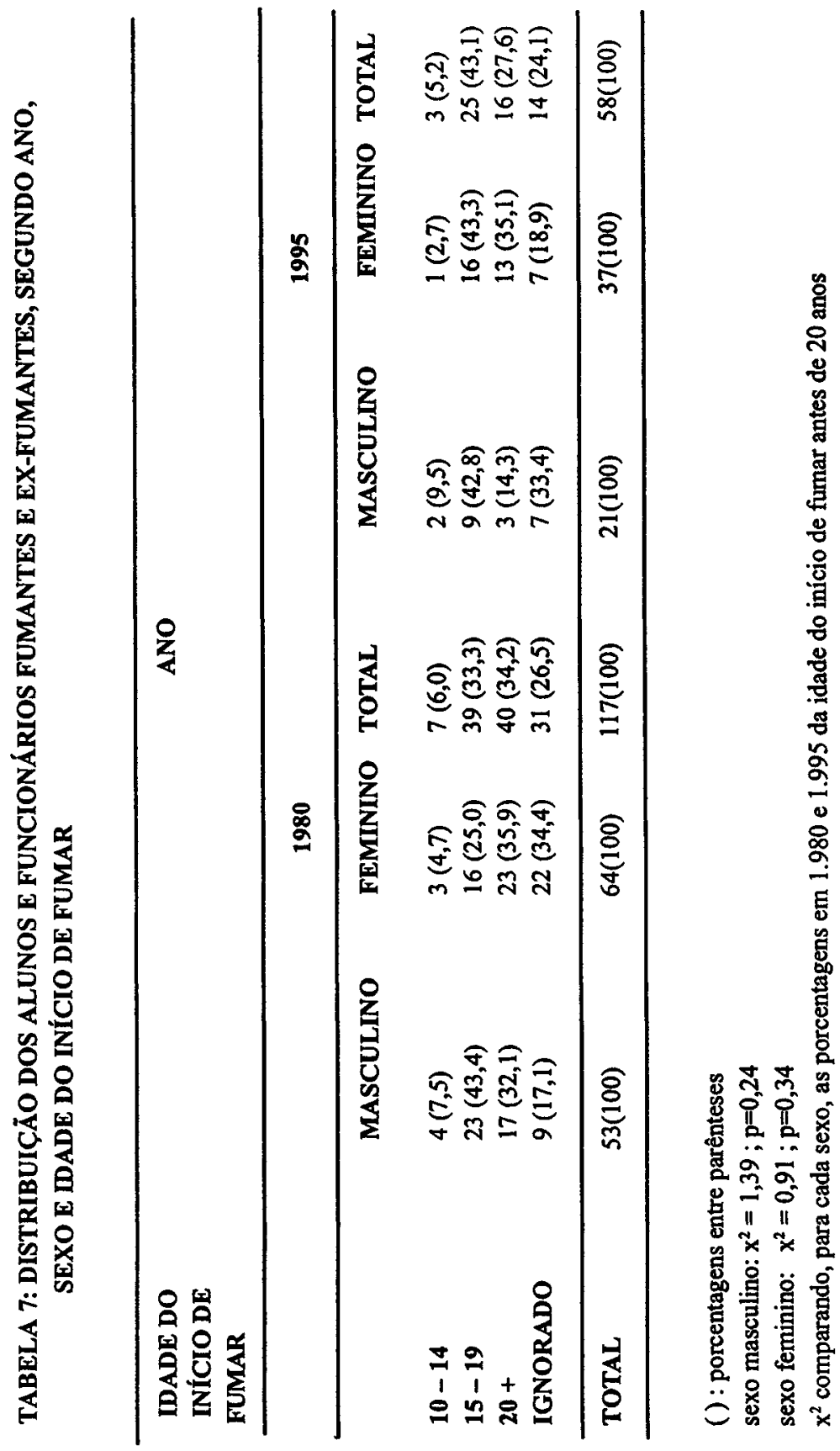




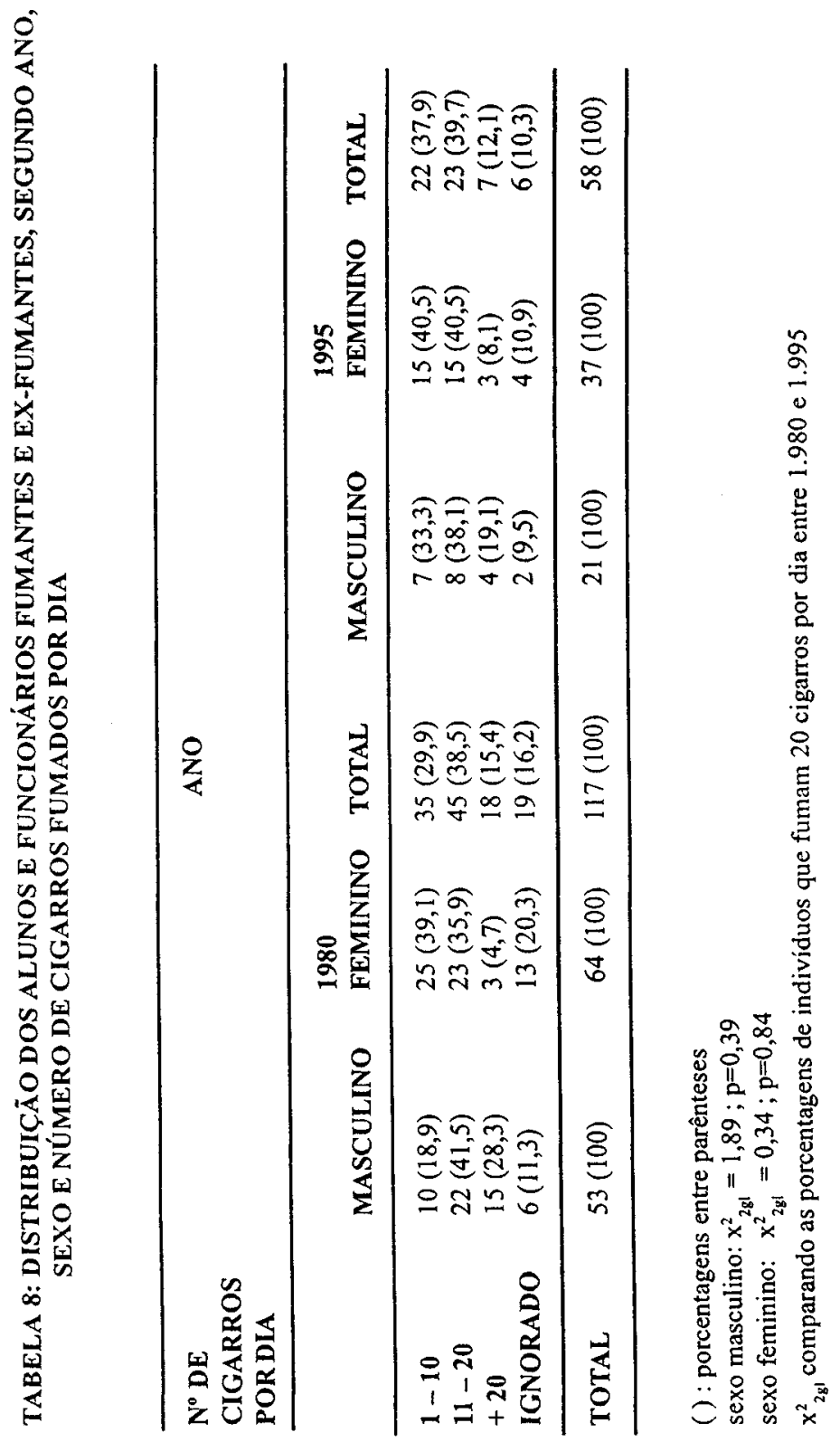




\section{SMOKING AMONG STUDENTS AND WORKS AT SCHOOL OF PUBLIC HEALTH/USP}

SUMMARY: This study evaluates the effects of a prevention program in smoking behavior. Two surveys about tobacco use among students and staff members of the School of Public Health of University of São Paulo who attended in the openning classes of the 1980 and 1995 academic years. In 1980 a total of 177 people were present (38,4\% men) and in 1995 a total of $128(29,7 \%$ men). From 1980 to 1995 the proportion of regular smokers fell from $56,9 \%$ to $26,3 \%$ in males and from $45,9 \%$ to $22,2 \%$ in females. Findings indicate that there was a higher decrease in the prevalence of regular smoking for younger subjects. Highly motivated to stop smoking were professionals of biosciences background, followed by social sciences and education. Less motivated were subjects of law background, who showed an increase in the prevalence of smoking. Most subjects informed that they have started smoking at the age group of 10 to 14 years, and that they were aware of the damage of tabacco smoking to health. The means considered as the most adequate for the divulgation dangers of the tobacco were TV and radio. More effective programs against smoking should be implemented in the schools of public health, in order to promote the decrease the number of smokers among their students and employees.

KEY WORDS: smoking, prevalence, health school.

Recebido: 24/11/98

Aprovado: 8/11/99 